\title{
Aproximación al trabajo alfarero e identidad en Laguna Blanca, Catamarca, Argentina.
}

\section{An approach to potting work and identity in Laguna Blanca, Catamarca, Argentina.}

doi https://doi.org/10.48162/rev.46.002

Valeria Elizabeth Espiro ${ }^{1}$

(D) https://orcid.org/0000-0001-8529-9707

\section{RESUMEN}

En esta contribución expondremos los resultados del análisis comparativo del proceso de trabajo alfarero correspondiente al modelado, en contextos domésticos de los siglos V al VII d.C., para dos aldeas de Laguna Blanca (Catamarca). Este estudio se inserta dentro de un objetivo mayor que busca reconstruir los procesos involucrados en la manufactura de los materiales cerámicos, para caracterizar el modo de trabajo alfarero y así dar respuesta al problema de la construcción de identidades sociopolíticas escindida o complementaria entre ambas aldeas. Desde el punto de vista teórico transitamos una arqueología de lo cotidiano y los modos de vida, que se completa metodológicamente desde la identificación de técnicas primarias y técnicas secundarias de manufactura a través de observaciones macroscópicas, submacroscópicas y microscópicas. Los datos obtenidos nos conducen a argumentar sobre la existencia de procesos de trabajo diferentes, dentro de modos de hacer compartidos entre ambos agrupamientos.

Palabras clave: procesos de trabajo, manufactura cerámica, identidad, Laguna Blanca.

\section{ABSTRACT}

This article presents the results of a comparative analysis of the modeling component of the potting process from the fifth to seventh centuries $\mathrm{AD}$ in domestic contexts in two villages in Laguna Blanca (Catamarca). This study is part of a larger project that seeks to reconstruct the processes involved in the manufacture of ceramic materials and characterize potters' ways of working, in order to address the

\footnotetext{
1 Escuela de Arqueología e Instituto Interdisciplinario Puneños, Universidad Nacional de Catamarca. valeriaespiro@unca.edu.ar
} 
problem of how the two villages' socio-political identities were constructed, as separate or complementary. From a theoretical point of view, we present an archeology of everyday life and ways of life. This uses the methods of identifying the primary and secondary production techniques through macroscopic, sub-macroscopic, and microscopic observations. The data lead us to present arguments about different and shared between the two villages in terms of work processes and ways of doing.

Keywords: work processes, ceramic manufacturing, identity, Laguna Blanca.

Recibido: 03/02/2021

Aceptado: 18/05/2021

\section{MARCO CONTEXTUAL Y CONCEPTUAL DEL ESTUDIO}

En esta contribución presentaremos los resultados de la descripción y el análisis de parte de la variabilidad tecno-morfológica de las vasijas cerámicas recuperadas en excavaciones de contextos domésticos de dos agrupamientos aldeanos del Bolsón puneño de Laguna Blanca, departamento Belén, provincia de Catamarca, Argentina (Figura 1).
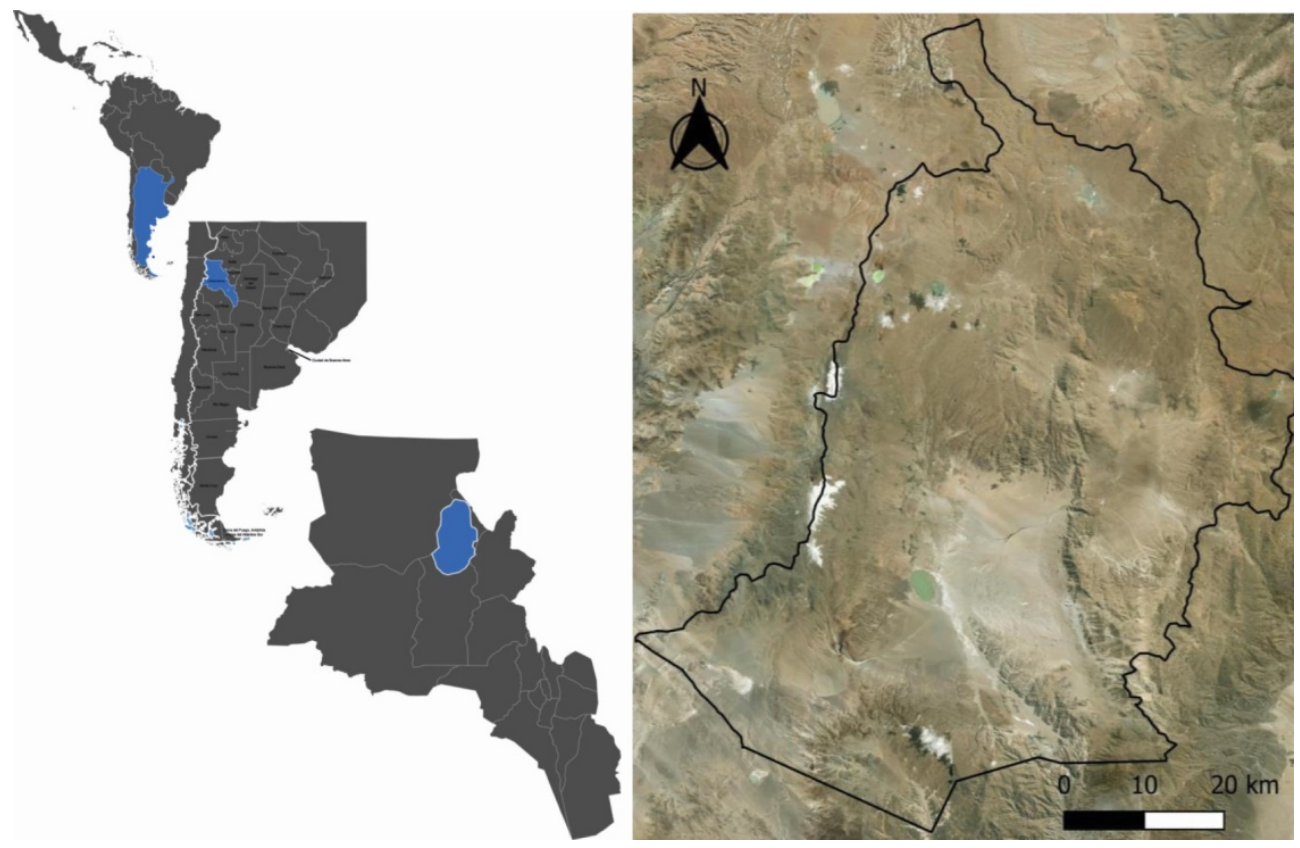

Figura 1. Ubicación del bolsón puneño de Laguna Blanca.

Este estudio se inserta dentro de un objetivo mayor de investigación que busca reconstruir los procesos involucrados en la manufactura de los materiales cerámicos (Acosta Ochoa, 1999, 2011), para caracterizar el modo de trabajo alfarero 
y así poder dar respuesta desde la producción cerámica al problema de la construcción de identidades socio-políticas escindida o complementaria entre ambas Aldeas. En tanto que los objetos - las vasijas cerámicas- son la objetivación del trabajo humano en un contexto social determinado, las decisiones tecnológicas y estilísticas en torno a los mismos tienen lugar en un proceso sujeto a las determinaciones históricas, económicas, políticas e ideológicas de la sociedad en su conjunto. Así podemos decir que los objetos cerámicos son referentes de atribución social, ya que desde la concepción misma del producto y a través del aprendizaje y la transmisión de una serie de gestos técnicos, en los conjuntos cerámicos quedan plasmadas ideologías y construcciones identitarias precisas (Dobres, 2000; Dobres y Hoffman, 1994; Padilla Fernández, 2017).

Los agrupamientos aldeanos, de los cuales provienen los materiales cerámicos analizados, se corresponden con la Aldea Piedra Negra y la Aldea Laguna Blanca, distantes unos pocos kilómetros entre sí y ubicados sobre el piedemonte oriental del Nevado de Laguna Blanca (Figura 2). A nivel de organización políticosocial hemos propuesto para las aldeas un Modo de Vida Comunitario Agrocéntrico (Delfino et al., 2009, 2015). Esta instancia de organización del conjunto de unidades domésticas campesinas representaría una respuesta de organización social para las actividades productivas, que entienden y viven el mundo bajo una metáfora agrícola.

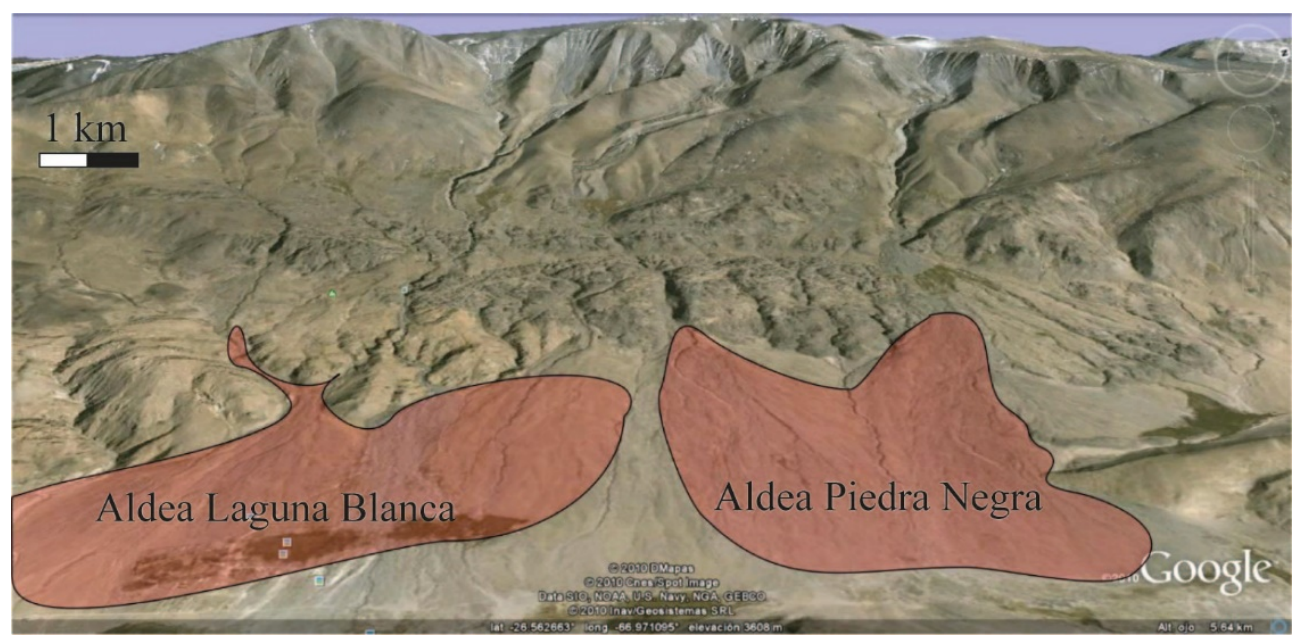

Figura 2. Panorámica de la ubicación de los agrupamientos Aldeanos.

La categoría de modo de trabajo tomada de Veloz Maggiolo (1984) se refiere a las diversas maneras concretas en que se cumple el proceso productivo de una sociedad dada, y supone una relación específica entre un conjunto de instrumentos de producción, una determinada organización del trabajo y una visión del mundo que 
la cohesione y le dé sentido. Debo aclarar que los modos de trabajo no son oficios, son las maneras de hacer en relación con una sociedad determinada y de acuerdo con las condiciones de su modo de vida (Vargas Arenas, 1985). La categoría de modo de trabajo nos permite reconocer la existencia de regularidades y ciclos en las actividades de una sociedad, a través de lo cotidiano, donde las actividades productivas, reproductivas e ideológicas se repiten rutinariamente (Acosta Ochoa, 1999). Pensar en lo cotidiano nos permite conectar con la reversibilidad colectiva y estructural de una constitución social (Giddens, 2011), es esta recursividad del saber compartido implicado en los procesos de trabajo lo que permite sostener una seguridad ontológica (las regularidades y ciclos en las actividades), que da por sentada una definición del mundo circundante, en especial de la vida cotidiana (Bialakowsky, 2018). Así, en la rutinización de las actividades cotidianas la conciencia práctica permea los modos de interpretar y (re)clasificar situaciones, contextos, actores y prácticas, a la vez que se vincula a la dinámica recursiva de lo social y la seguridad ontológica del mundo cotidiano (Bialakowsky, 2018). En el caso de la alfarería, esta dinámica adopta la forma de la rutinización de los procesos de trabajo sobre la base de los saberes mutuos y la emergencia de ciertos principios estructurales vinculados a los modos de hacer en un tiempo y espacio. Siguiendo a Giddens (2011), son estos saberes mutuos los que, distribuidos de forma heterogénea en una sociedad, permiten aplicar reglas o pautas (informales y formales) y representaciones para interpretar y reclasificar el saber hacer de forma diestra y práctica. Al poner en juego este trasfondo de saber mutuo en los modos de trabajo alfarero, estos se vuelven reflexivos y negocian los diversos procesos de estructuración de las prácticas de cada alfarero en particular, como las formas de acceso a ciertos "recursos o materias primas" o a "información referida a las técnicas", atravesados por las asimetrías en la distribución de recursos y en los grados de legitimidad de jerarquías y saberes dentro del mismo grupo social. Estos procesos implican la estructuración de tradiciones espacio-temporales, las cuales regionalizan las prácticas a partir de distintos atributos, visibilidades e invisibilidades para cada una de estas regiones centrales o periféricas (valle, aldea, base residencial, etc.), lo cual nos permite identificar tradiciones alfareras particulares.

Así tenemos que un modo de trabajo integra, a su interior, la organización de los procesos necesarios para la relación del individuo con su objeto de trabajo. Metodológicamente, un modo de trabajo puede ser inferido mediante el ciclo de actividades definido a partir de los conjuntos artefactuales que establecen entre sí una relación necesaria (Acosta Ochoa, 2011).

Los procesos de trabajo que es posible identificar y que integran el modo de trabajo alfarero son: 1 . Procesos de extracción de las materias primas, en los que se incluyen los procesos de producción de las materias primas principales o auxiliares. 
2. Procesos de preparación, que incluyen molienda, hidratación de la mezcla (arcilla y engobe) y maduración. 3. Proceso de modelado. 4. Tratamientos de superficie. 5. Decoración precocción. 6. Cocción. 7. Decoración poscocción. 8. Acabado.

El orden expuesto en los procesos anteriores es preferente, pero no es el orden necesario de todos los modos de trabajo singulares. En el presente estudio nos centraremos en la descripción del proceso de modelado de los objetos cerámicos, ya que, dentro del trabajo alfarero, constituye uno de los más relevantes en cuanto al conjunto de acciones, gestos y técnicas que involucra (Balfet et al., 1992; Rye, 1988). Este proceso es realizado cuando la pasta cerámica se halla en estado plástico, y así las propiedades de la arcilla en ese momento posibilitan que se pueda variar la forma final del objeto, lo que requiere que se conjuguen los conocimientos y la experticia del alfarero o de la alfarera con las propiedades de las materias primas empleadas.

Consideramos que el tipo de conocimientos que podemos obtener del análisis del proceso de modelado involucra tanto a las técnicas de manufactura como a la secuencia de las acciones y gestos que intervienen en la labor alfarera (Balfet $e t$ al., 1992; Rice, 1987; Rye, 1988).

En cuanto al modelado, si bien existen varios modelos o secuencias propuestas, en esta investigación se tomó como referencia la propuesta de Rye (1988), que distingue entre técnicas primarias y técnicas secundarias de manufactura. Las primarias son aquellas que se aplican para que la pasta sea modelada hasta lograr una primera forma base: comúnmente los contenedores cerámicos se elaboran por partes, sobre todo aquellos de dimensiones medianas y grandes. Las técnicas secundarias son identificadas con aquellas que se realizan cuando la pasta se encuentra en estado "cuero" o seco (previo a la cocción), pero una vez que la forma del objeto ya está conformada y las proporciones de las diferentes partes ya están definidas. Para complementar esta propuesta se siguieron estudios etnográficos y experimentales tales como los de Arnold (1994), De la Fuente (2011), García Rosselló (2011), entre otros.

En este trabajo se siguió un camino metodológico que combinó tres instancias analíticas: macroscópicas, submacroscópicas y microscópicas. Las observaciones macroscópicas se realizaron a ojo desnudo y con ayuda de lupa de mano sobre la totalidad de la muestra de los materiales cerámicos procedentes de ambos sitios (PIN 02 n=5037 y LB19 n=1400), las cuales permitieron el agrupamiento en familias de fragmentos (Orton et al., 1997). Para la confección de las familias se consideró el supuesto de que se agrupan todos los fragmentos pertenecientes a una misma vasija. Los criterios utilizados para asumir que potencialmente los tiestos podrían pertenecer a una misma pieza fueron las características de la pasta (color y textura), el tratamiento de superficie, los espesores de las paredes y la forma. 
El nivel de estudio submacroscópico implicó la observación a bajos aumentos de la superficie de los fragmentos, así como de la pasta mediante la realización de fracturas frescas. Empleamos una lupa binocular estereoscópica marca Arcano, con aumentos de 20x y 40x, y en todos los casos con el uso de luz halógena incidente. Se seleccionó una muestra representativa de todas las familias de fragmentos para PIN $02(n=1019)$ y de LB $19(n=696)$. Para el registro de las observaciones se confeccionó un código analítico para familias de fragmentos y su correspondiente ficha alfanumérica, diseñada e ingresada en Excel, lo cual facilitó su posterior tratamiento estadístico. Las observaciones realizadas incluyeron más información que la empleada en este trabajo, tales como datos generales del fragmento, relacionados al proceso de manufactura (datos de la pasta, técnica de trabajo de la pieza, técnicas de decoración, tipo de cocción, tratamiento de superficie), datos referidos a efectos de uso, evidencias de mantenimiento, de reutilización y/o reciclado, efectos de depositación.

Para la instancia microscópica, el objetivo central del análisis fue profundizar las observaciones realizadas en las etapas previas, sobre todo características tecnológicas de la pasta en cuanto a la matriz y las inclusiones. Se trabajó con muestras representativas de las variabilidades observadas en lupa binocular de todas las familias (para PIN $02 n=35$ y para LB $19 n=12$ ) y para prestar especial atención al proceso de manufactura se realizaron cortes de secciones paralelas a la pared de la pieza. Como con el estudio submacroscópico, se confeccionó una ficha de análisis para cortes delgados cerámicos y se hizo uso de microscopio petrográfico con luz paralela y polarizada, para la muestra de PIN 02 se trabajó con un microscopio petrográfico trinocular DRU3-MEOPTA (con objetivos de de 40x, 100X y 250x) y para la muestra de LB 19 se utilizó un microscopio trinocular BIOTRAZA modelo XP-148PLT (con objetivos de 40x, 100x y 250x).

\section{LOS CONTEXTOS DOMÉSTICOS}

Para ubicar nuestro análisis brindaremos una breve descripción de los contextos domésticos de los cuales proviene el material analizado.

La base residencial Piedra Negra 02 (PIN 02), se ubica en el centro de la Aldea Piedra Negra, está integrada por 9 recintos subcirculares adosados y distribuidos alrededor de 3 recintos mayores de planta cuadrangular, y por 3 recintos mayores de planta cuadrangular (Espiro, 2008. Figura 3). 


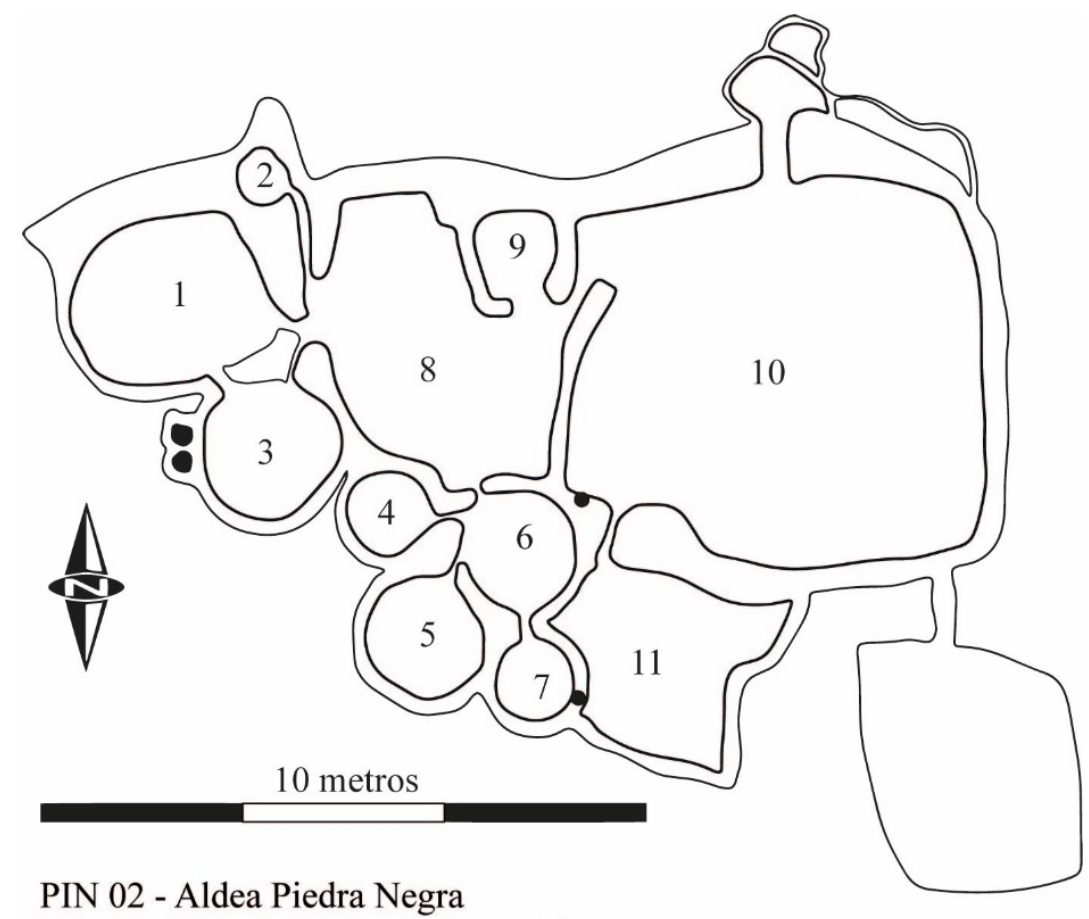

Distristo Laguna Blanca Dpto. Belén - Catamarca

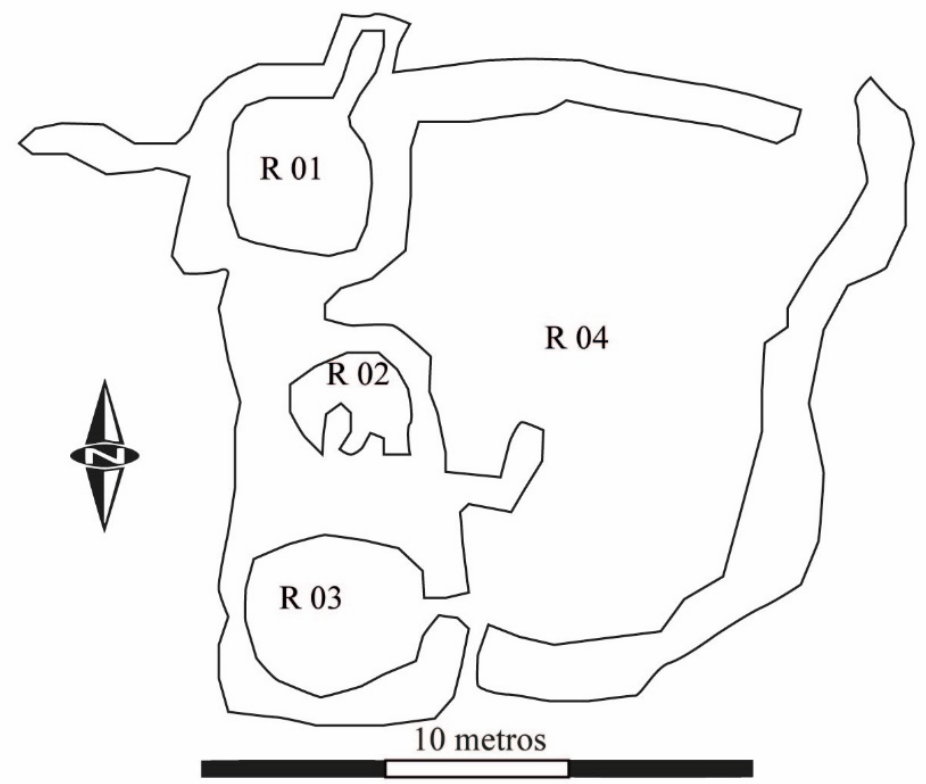

LB 19 - Aldea Laguna Blanca

Distristo Laguna Blanca - Dpto. Belén - Catamarca

Figura 3. Planos de las bases residenciales Piedra Negra 2 (arriba) y de LB 19 (abajo). 
En esta unidad habitacional se han excavado estratigráficamente por área abierta cuatro recintos y se empleó en este análisis el material proveniente de uno de ellos (recinto 02, antes denominado A). Entre las evidencias artefactuales, recuperadas en el recinto 02 de PIN 02, se destacan varios objetos de metal (en cobre y oro), además de artefactos en hueso, cientos de restos óseos faunísticos, gran cantidad de material lítico y más de cinco mil fragmentos cerámicos (Delfino, 1999; Delfino et al., 2007).

Las características presentes en los materiales recuperados, la presencia de una estructura de combustión, un muro deflector y de varios instrumentos de molienda, nos llevaron a interpretar a este espacio intramuros como destinado a la realización de actividades domésticas. Se consideró que en el mismo se llevaba a cabo la preparación, la cocción y el consumo de alimentos, así como otras actividades productivas y reproductivas.

La Base residencial LB 19 (Figura 3), se ubica en el sector central de la Aldea Laguna Blanca, está integrada por dos recintos circulares, conectados por un tercer recinto de forma subcircular, y a su vez estos tres recintos se unen a un recinto mayor de forma cuadrangular que puede ser interpretado como un patio. En LB 19 se excavaron por área abierta dos recintos y parcialmente el patio. El material cerámico objeto de análisis de este trabajo proviene del recinto 03 . Si bien la estratigrafía y los restos materiales recuperados en el recinto 03 de LB 19 se encuentran bajo análisis, de manera preliminar podemos mencionar el hallazgo de artefactos en hueso, cientos de restos óseos faunísticos, más de mil restos de material lítico (abundantes lascas e instrumentos confeccionados en diversas materias primas) e instrumentos de molienda y unos 1400 fragmentos cerámicos; también se encontraron evidencias de una estructura de combustión.

Los recintos excavados tanto en PIN 02 y LB 19 fueron construidos con paredes de roca disponible en el mismo piedemonte, según hiladas simples y con aparejos rústicos. Pudimos identificar que la construcción de los recintos implicó la remoción de tierra para lograr el nivel negativo de su interior (Delfino, 1999, 2005). Se cuenta con fechados radiocarbónicos para ambos recintos, los cuales fueron realizados sobre carbón recogido de las estructuras de combustión, para LB 19 R03 $1470 \pm 40{ }^{14} \mathrm{C}$ AP (LP 2589) y para PIN 02R2 $1260 \pm 70{ }^{14} \mathrm{C}$ AP (LP 1306) (Delfino et al., 2015).

\section{LOS PROCESOS DE TRABAJO ALFARERO}

Ahora bien, para poder determinar los procesos de trabajo involucrados en la producción alfarera de ambos recintos, en una primera instancia necesitamos realizar una caracterización tecnológica de los objetos cerámicos. En este caso nos 
centramos en la identificación de las técnicas y maneras de hacer empleadas en el proceso de modelado de las cerámicas de cada conjunto.

Nuestro universo de análisis fue de 5037 fragmentos cerámicos para el PIN 02 R2 y de 1400 fragmentos para LB 19 R03. En primera instancia y a partir de atributos observables macroscópicamente realizamos el agrupamiento de fragmentos por familias, y consideramos a cada una como una vasija cerámica (Orton et al., 1997). Dentro del total del conjunto analizado pudimos conformar 38 familias de fragmentos para el PIN 02 R2 y 27 para LB 19 R03. También se identificaron instrumentos cerámicos, en su mayoría alisadores (Figura 4), 14 para PIN 02 R2 y 17 para LB 19 R03.

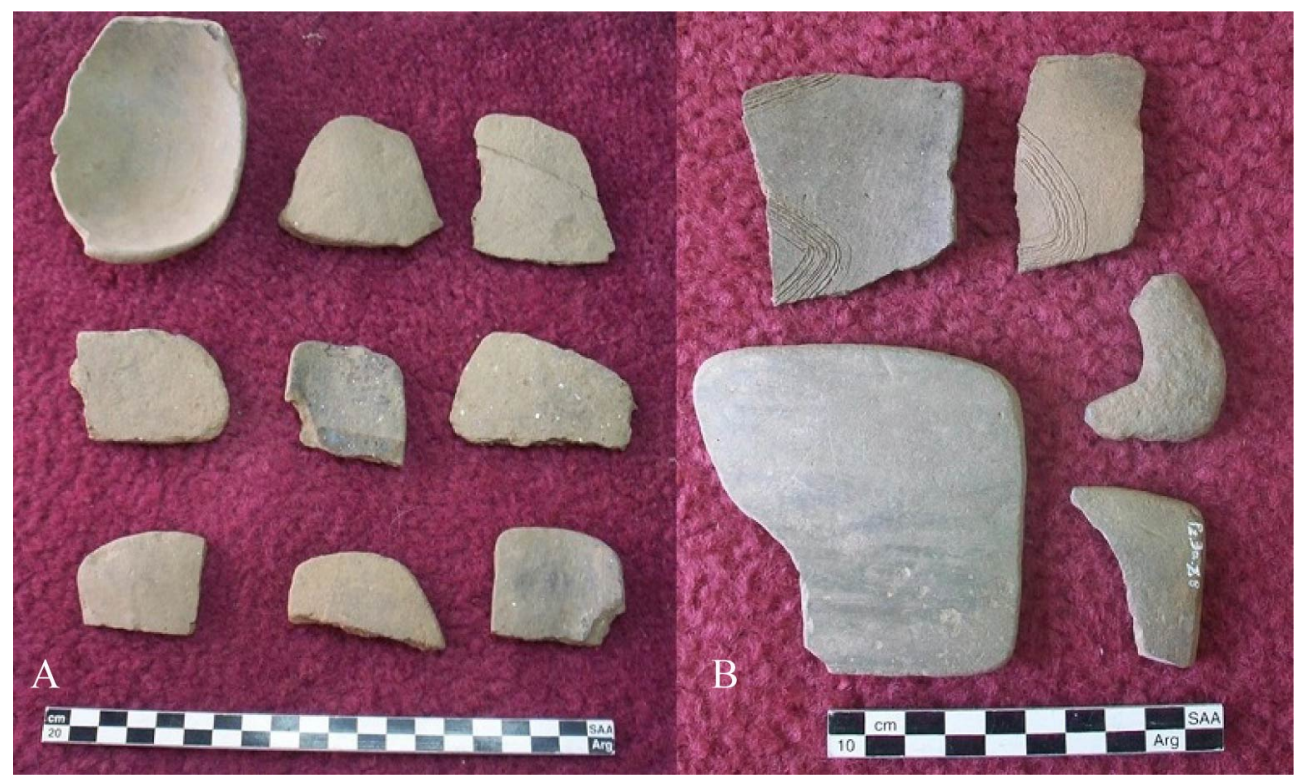

Figura 4. Alisadores cerámicos hallados en ambos sitios A) LB 19 y B) PIN 02.

Para la identificación de las formas se siguieron los lineamientos de Balfet et al. (1992), de esta manera se registraron las formas de vaso/jarra, puco/escudilla y olla en los dos recintos (Figura 5). Para PIN 02 R2 cuatro familias corresponden a la forma de vaso/jarra, 18 a la forma de puco/escudilla y 10 a la forma de olla. Para LB19 R03, tres familias se corresponden a la forma de vaso/jarra, 12 a pucos y 12 a ollas. De acuerdo a estos resultados podemos señalar que más allá de la diferencia numérica en la cantidad total de fragmentos, al agruparlos en familias esta no se vuelve tan amplia. 
Valeria Elizabeth Espiro 2021. Aproximación al trabajo alfarero e identidad en Laguna Blanca, Catamarca ...
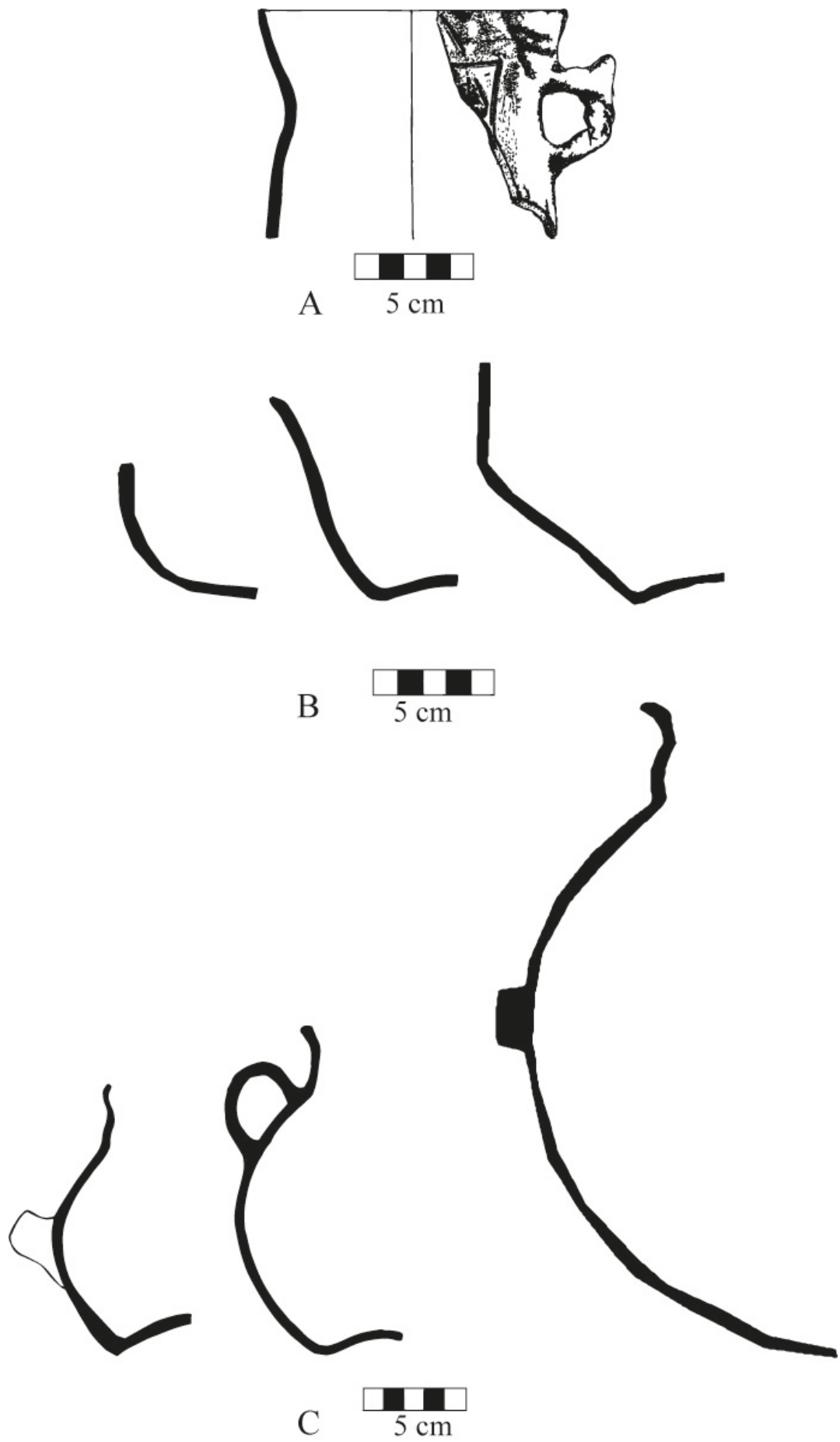

Figura 5. Variabilidad de formas identificadas en ambos conjuntos; A) vaso o jarra; B) perfiles de pucos o escudillas; $C$ ) perfiles de ollas. 
En cuanto a las técnicas identificadas para el trabajo de la pieza se tomaron las propuestas metodológicas de Balfet et al. (1992) y Rye (1988) y nos centramos en las técnicas de manufactura primarias (tales como rodeteado, torneado, laminado, etc.) y algunas secundarias (tales como la extracción o el ahuecado) que hacen al modelado del cuerpo sin considerar aquellas que implican el decorado de la pieza. Así fue que se han observado algunas diferencias entre ambos recintos.

Para PIN 02 R2 se identificaron las siguientes técnicas primarias: $66 \%$ de rodete, $11 \%$ laminado (empleo de placas o panchas) y $11 \%$ modelado; y un $12 \%$ correspondió a la técnica secundaria de extracción (la cual implica la remoción de pequeñas cantidades de pasta para lograr uniformidad en el espesor de las paredes previa a la aplicación de técnicas decorativas). Para LB19 R03 se contempló el empleo de las siguientes técnicas primarias: 40\% de rodete, 40\% laminado, 7\% modelado y rodete y $7 \%$ modelado.
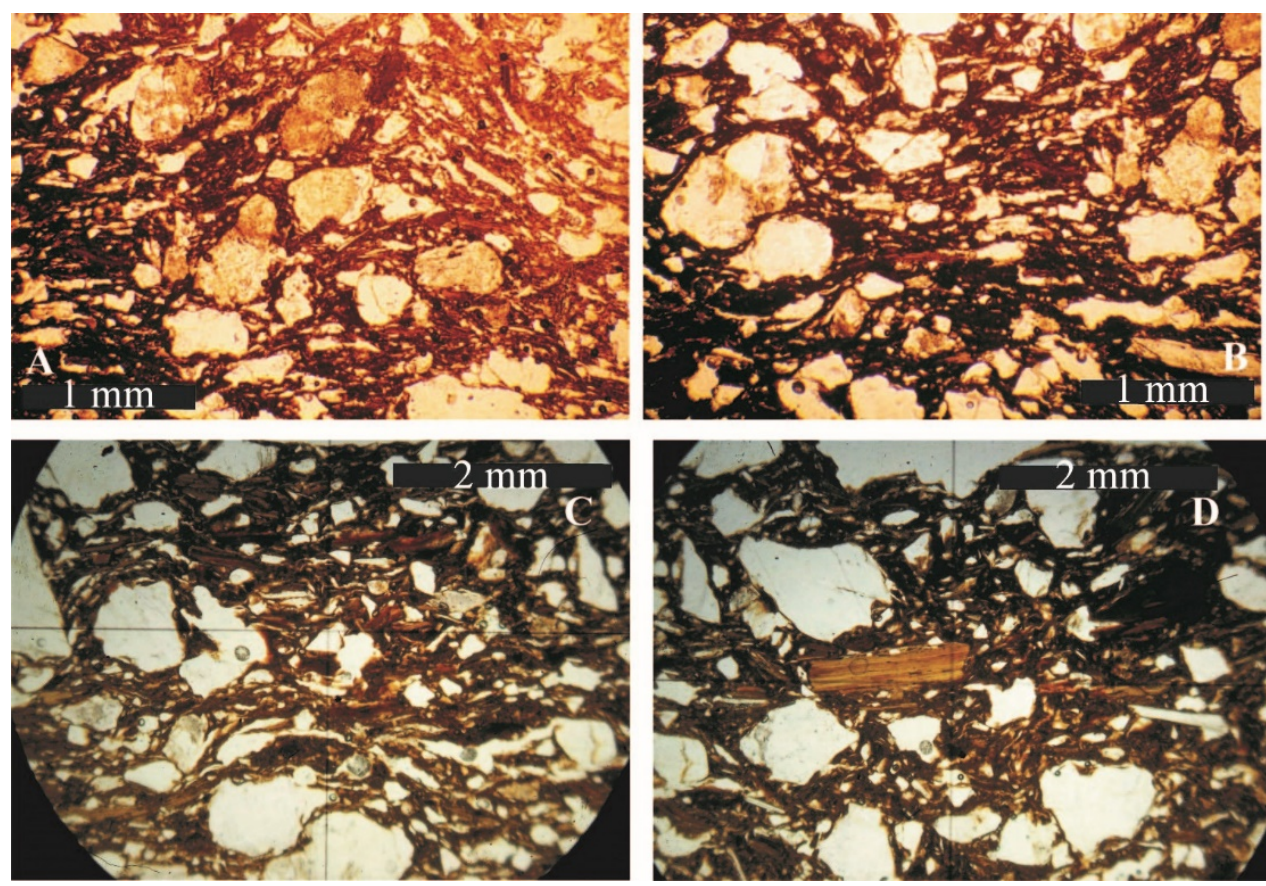

Figura 6. Fotomicrografía de cortes delgados paralelos que registran evidencias de técnicas primarias, posiblemente rodete (observación con nicoles paralelos). A) cuerpo de puco de PIN 02 y B) cuerpo de olla de PIN 02; C) cuerpo de olla de LB 19 y D) cuerpo de puco de LB 19. Se observa orientación paralela y subparalela de antiplásticos y cavidades elongadas - probable utilización de técnica de rodeteado (láminas delgadas paralelas).

La identificación de la técnica de rodete se logró gracias a la observación de los siguientes indicadores a escalas macroscópica y submacroscópica: 1) orientación 
horizontal de los antiplásticos en las caras superficiales y orientación circular de partículas en las bases en cara interna; 2) diferencias de espesor en el perfil horizontal de la pared, tanto en el interior como el exterior de la pieza -entre la unión de los rodetes-; 3) quiebres selectivos paralelos en la zona de unión entre rodetes; y 4) marcas de la unión defectuosa entre rodetes. En cuanto a las observaciones microscópicas, en cortes delgados paralelos de fragmentos las cavidades y los antiplásticos se registraron elongados (y en menor medida esféricos), con orientación fluidal o paralela y subparalela, situación que hemos tomado como evidencia de la técnica de rodeteado (Figura 6).

En cuanto a la segunda técnica más registrada, el laminado (levantado mediante láminas, planchas o placas de masa arcillosa), se observaron a nivel macroscópico y submacroscópico fracturas laminares en los fragmentos indicativas de esta, así como grietas y fracturas surgidas en el punto del engrosamiento exterior en donde se aplicó una lámina para el ensanchado del cuerpo o en la unión del cuerpo y la base.

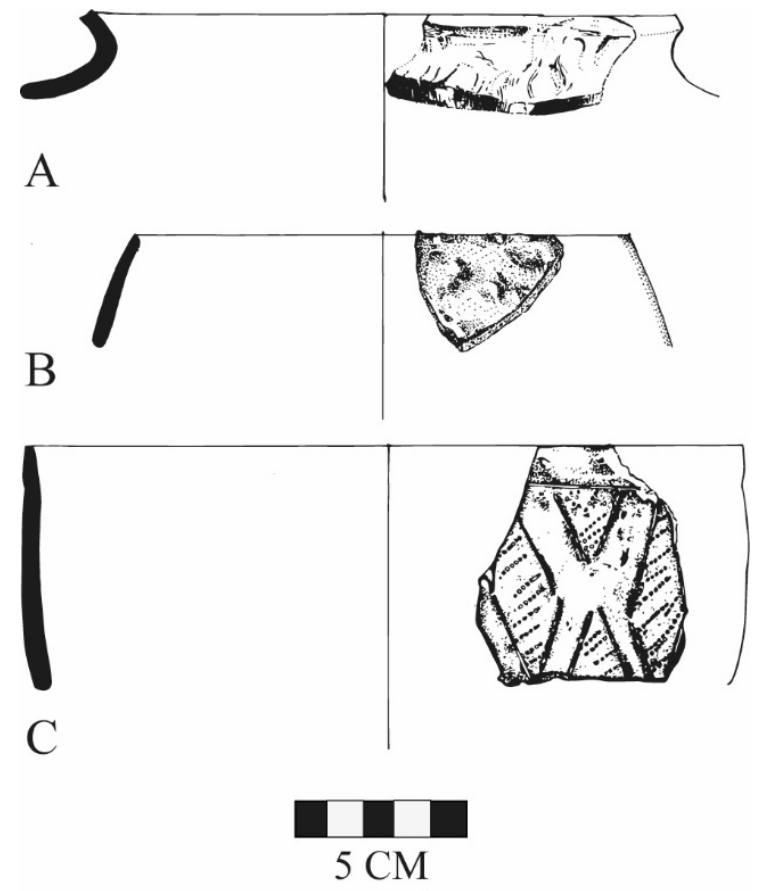

Figura 7. Variabilidad de forma del ángulo del borde y forma labio registradas; A) olla de PIN 02 con ángulo obtuso de borde y forma de labio recto, B) puco de PIN 02 con ángulo agudo y labio biselado, C) puco de PIN 02 de ángulo recto y labio convexo. 
En cuanto al modelado, se identificó mayormente en las bases, las cuales exhibieron uniformidad en la pasta sin diferenciación en su corte transversal, aleatoriedad en la distribución y ordenación de los antiplásticos, ondulaciones verticales e impresión de huellas digitales.

En lo referente a la forma del ángulo del borde, en términos generales sin discriminar por forma de los contenedores cerámicos se pudieron observar las siguientes proporciones para PIN 02 R02: $54 \%$ recto, $26 \%$ obtuso, $17 \%$ agudo y $3 \%$ recto y agudo; y para LB 19 R03: 50\% recto, $42 \%$ obtuso y $8 \%$ agudo (Figura 7 ).

Sobre la forma del labio, podemos indicar que la mayor diferencia se observa en la abundancia de bordes rectos (50\%) y dobles biselados (10\%) en LB 19 R03, mientras que para PIN 02 R02 la mayoría de los bordes tienen labios convexos (60\%) (Figuras 7 y 8 A y C).

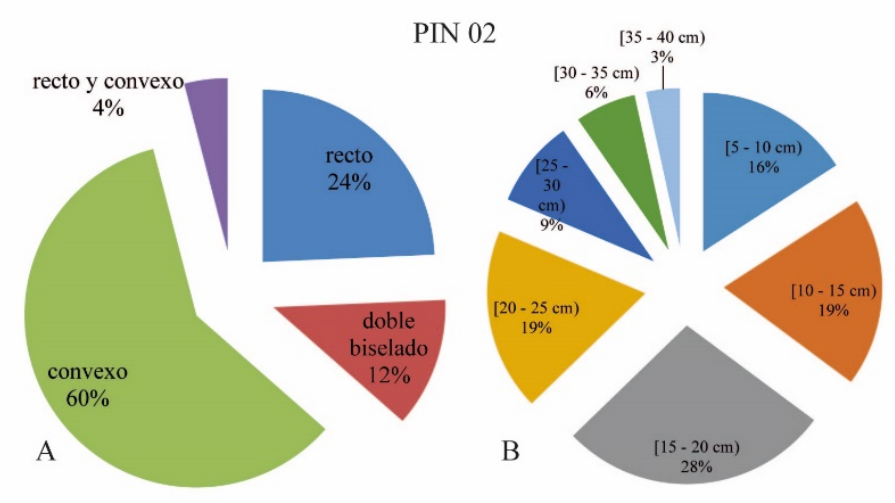

LB 19
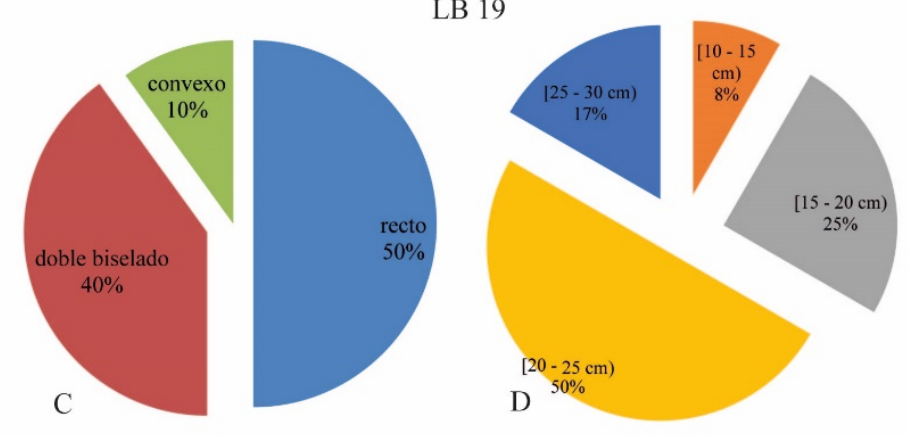

Figura 8. Comparación de forma del labio: A) PIN 02 y C) LB 19; y diámetro de borde de las vasijas: B) PIN 02 y D) LB 19. 
Si tomamos en cuenta la variable del diámetro de la abertura o boca de los contenedores cerámicos, vemos que PIN 02 R02 posee mayor diversidad, presentando a su vez vasijas de grandes dimensiones, sobre todo de ollas subglobulares que superan los $30 \mathrm{~cm}$ de diámetro (Figura 8 B y D). Consideramos que esta variabilidad es relevante en términos del proceso de modelado, pero también para poder dilucidar aspectos vinculados al uso y consumo de los materiales cerámicos, por lo que profundizaremos las relaciones entre estas variables en futuros estudios de los conjuntos cerámicos.

En cuanto a las asas podemos mencionar, en primer lugar, que en ambos sitios se identificaron para las formas de jarra o vaso y ollas; mientras para LB 19 solo se encontraron asas en cinta, con posición vertical, unidas al cuerpo mediante una inserción en forma de remache, para PIN 02 R02 se observó una mayor variabilidad de formas de asas (Figura 9), a pesar de que hubo ejemplares en donde no se pudo determinar la posición o inserción.
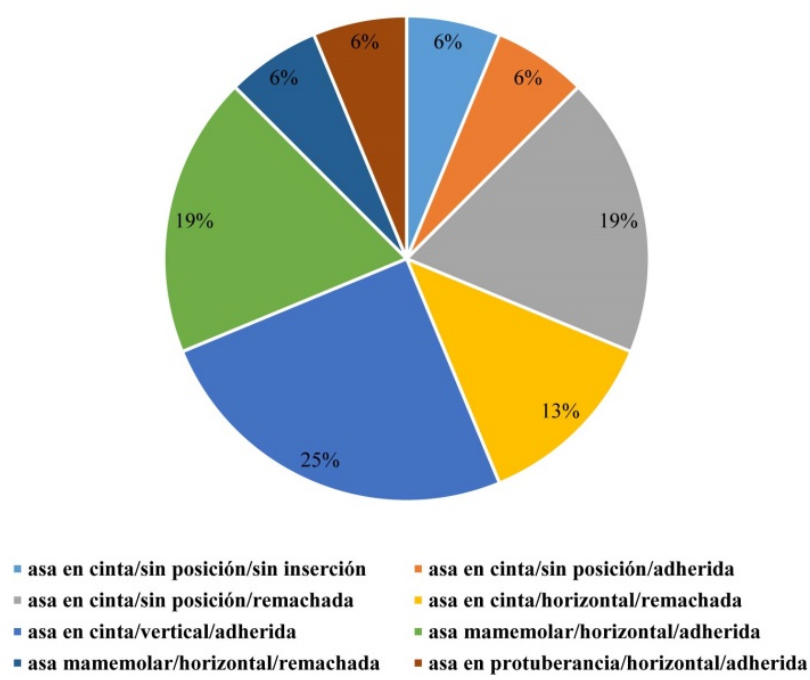

Figura 9. Variabilidad de tipos de asas identificadas en PIN 2

En cuanto a las bases, podemos decir que se contabilizaron muy pocas dentro de todo el conjunto, no obstante, se observaron tendencias semejantes en ambos recintos en lo que hace a la forma y al tamaño de las mismas (Figura $10 \mathrm{~A}$ y C). En cuanto a las que se pudo identificar la forma de contenedor cerámico, la mayoría de las bases se correspondían a formas de puco o escudilla. 
En cuanto al tipo de cocción, si bien las diferencias son mínimas entre ambos recintos, se puede observar para LB 19 R03 más familias de fragmentos de cocción reductora en comparación con PIN 02 R2 (Figura 10 B y D).

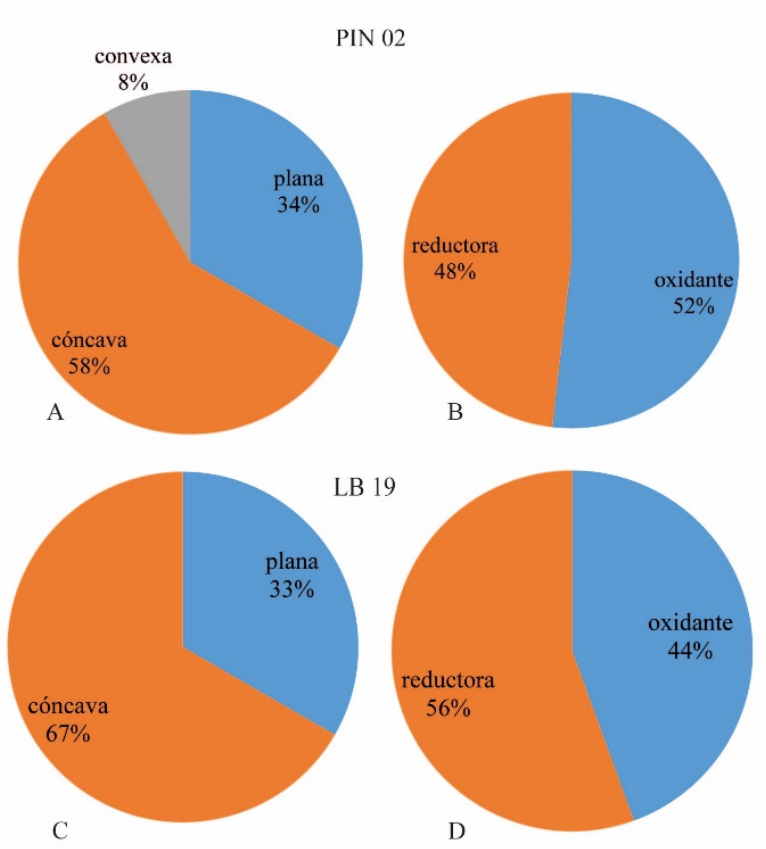

Figura 10. Comparación de las formas de las bases: A) PIN 02 y C) LC 19; y tipos de cocción: B) PIN 02 y D) LB 19.

\section{NOTAS SOBRE IDENTIDAD Y TRABAJO ALFARERO}

Podemos plantear como resultados preliminares de esta comparación del proceso de modelado de las vasijas cerámicas provenientes de PIN 02 R2 y LB 19 R03 algunas diferencias significativas en cuanto a las técnicas aplicadas. Señalamos la preponderancia de la técnica de rodeteado en las vasijas de PIN 02, mientras que en LB 19 se observaron las mismas proporciones para las técnicas por rodete y por laminado. Así también se identificaron mayoritariamente labios de forma convexa en PIN 02, mientras que las formas más abundantes en LB 19 fueron rectas y doble biselado. Entre las vasijas de PIN 02 se registró mayor variabilidad en las formas, posición y tipo de inserción de las asas. En lo que refiere a los tamaños y diámetros también se pudo registrar la presencia de mayor variabilidad de tamaños en PIN 02, con la presencia de vasijas cerámicas de grandes dimensiones. 
Ahora bien, ¿qué interpretaciones podemos derivar a partir de estos datos preliminares del modo de trabajo alfarero, que resulten significativas para dar respuesta al problema de la existencia de identidades socio-políticas escindidas o complementarias entre ambas Aldeas?

Antes de avanzar con nuestras argumentaciones, podemos tomar otra línea de evidencia brindada por el análisis de los espacios productivos agrícolas. Se sugiere que durante el primer milenio estos espacios en los agrupamientos aldeanos estudiados pudieron articularse complementariamente, pero los mecanismos de gestión productiva recaerían preponderantemente en los pobladores que habitaban la Aldea PIN (Delfino et al., 2010). Es decir, que podríamos suponer que los habitantes de ambas aldeas podrían poseer una identidad socio-política compartida y complementaria.

Podemos considerar la presencia de alisadores cerámicos en ambas bases residenciales (Figura 4) como evidencias de que, al menos, algunas etapas del proceso de trabajo cerámico se llevaban a cabo en ellas y así suponer que la producción alfarera no era un proceso centralizado, al menos espacialmente, en una de las aldeas. Así, parte de las variaciones observadas podrían deberse a esta diferente localización. Si consideramos que parte de la secuencia de trabajo alfarero, como es el modelado, se llevaba a una escala doméstica, la transmisión del conocimiento de las técnicas y operaciones cerámicas es un asunto del grupo social que habita en el mismo espacio, por lo que se aprende en la casa como parte del resto de las actividades de la vida cotidiana (Vidal y García Rosselló, 2009). En este sentido, en contextos de producción doméstica, el conocimiento técnico se trasmite generación tras generación, a través de un aprendizaje dentro del grupo conviviente. Desde la infancia, el alfarero o la alfarera aprende el trabajo mediante "el saber hacer" trasferido por un/a adulto/a del grupo conviviente, que a la vez aprendieron de otros, y así sucesivamente. Podemos agregar que en el aprendizaje de las técnicas de modelado existe un control social sobre determinados gestos técnicos (García Rosello, 2011), ya que la transmisión de estos conocimientos se basa en la transferencia de los esquemas físico-motores necesarios para adquirir el saber hacer relacionado con el modelado de la cerámica.

No obstante, este proceso debe concebirse a nivel social, en tanto que lo que se trasmite no es únicamente el saber hacer del experto, sino que engloba el conocimiento tecnológico que tiene el grupo social al que pertenece. Así vemos que se trasmite un conocimiento tecnológico social e identitario (Figura 11). 


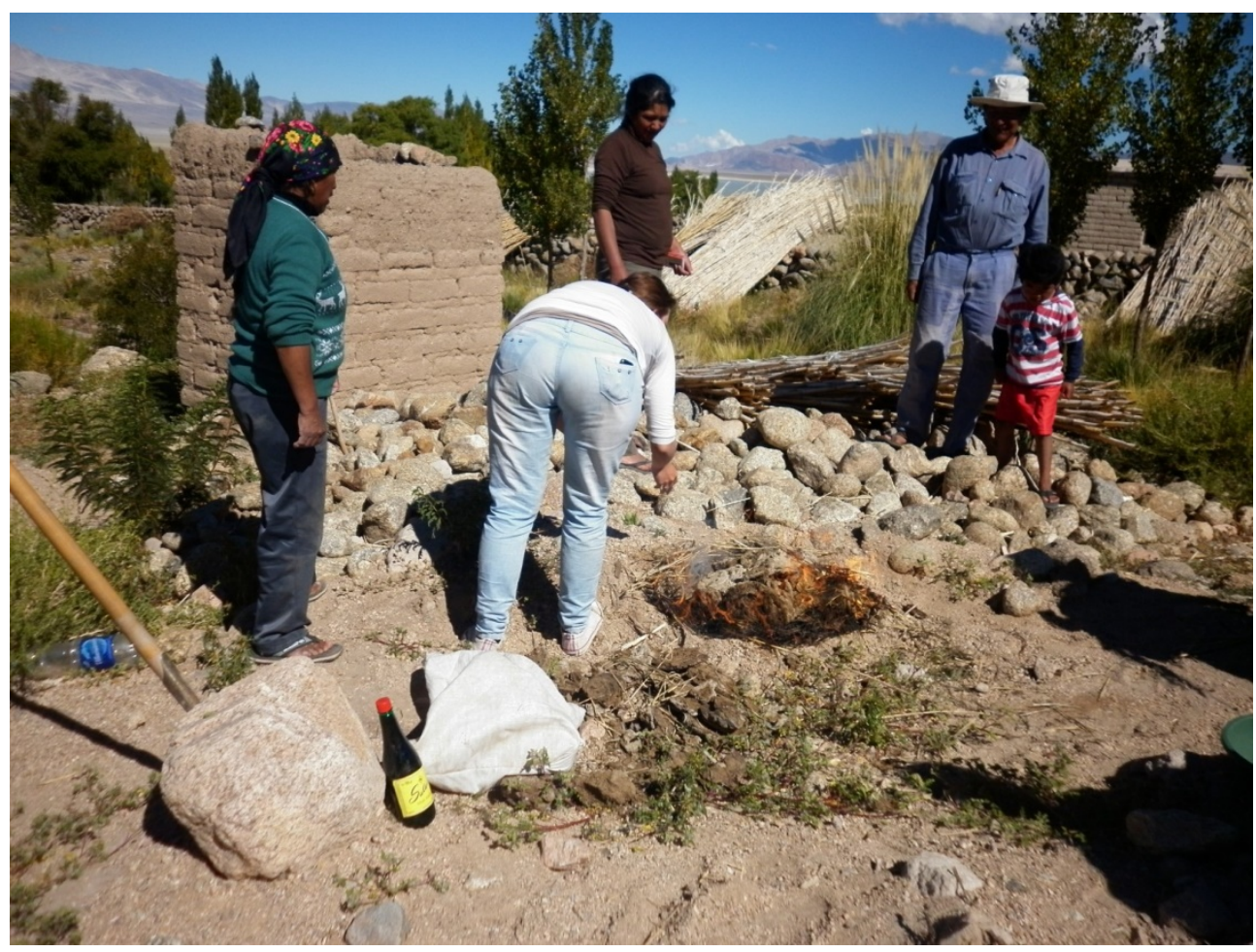

Figura 11. Cocción actual de alfarerías en la comunidad indígena de Corral Blanco, Laguna Blanca, donde se observan varios miembros y generaciones de la familia de la Cacique Doña Rosa Salgado y del comunero Don Felix Pachado participando del proceso

Si consideramos el Modo de Vida Comunitario Agrocéntrico, este proceso de aprendizaje dentro del ámbito doméstico implicaría la transmisión de conocimientos técnicos referidos al modelado de la cerámica, pero que combinaría con otros aprendizajes tecnológicos y sociales, vinculados al mundo vivo que se cría y deja criarse; saberes sobre la prácticas gastronómicas, agrícolas, pastoriles, la caza y los textiles, entre otros. Ahora bien, si las maneras de hacer cerámica se comparten y transmiten socialmente como cualquier otro aspecto de la cultura de una sociedad, podemos suponer que las diferencias observadas en la manera de realizar los labios de las vasijas, o en cómo colocar y hacer las asas, etc., estarían respondiendo a diferencias en la transmisión de conocimientos más que a causas de índole funcional. Podríamos buscar respuestas a esto, suponiendo que los mecanismos de transmisión se vinculen a relaciones sociales de parentesco (consanguíneo o de crianza), más que a cuestiones de género o generacionales; y otro aspecto importante es que mayormente este tipo de conocimiento se transmite de forma verbal, pero también por imitación, demostración y autoaprendizaje (Varela Guarda, 2002). Esto último en el contexto de la rutinización de las prácticas del modo de trabajo alfarero, en la 
construcción de lo cotidiano, se vuelve en una condición imprescindible de la vida tanto individual como colectiva-comunitaria. Lo cotidiano posibilita en la rutinización la transmisión de conocimiento, mediado por la repetición y también por la creación y recreación (Bialakowsky, 2018); lo que explica que, dentro de un mismo grupo social, los alfareros escojan entre un repertorio amplio de recursos conocidos aquellas técnicas y procedimientos de trabajo con los que se sienten más conformes desde su experticia.

Para finalizar y esperando poder profundizar nuestras investigaciones, no podemos cerrar con algún aserto medianamente concluyente; no obstante, nos planteamos que, frente a las diferencias observadas en los procesos de modelado, habría que preguntarse si serían indicativos de procesos de trabajo sustancialmente distintos que sugieran una alineación con modos de hacer alfareros diferentes para ambas Aldeas, a pesar de que sus pobladores puedan compartir o complementarse en otros aspectos culturales e identitarios.

\section{AGRADECIMIENTOS}

A los evaluadores del trabajo, que realizaron aportes muy valiosos para mejorar su calidad científica; así como a las editoras por sus excelentes sugerencias, paciencia y compromiso. A mis compañeros de equipo de INIP-UNCA con los cuales debatimos y compartimos muchas ideas en las horas de campo y gabinete, así como a los miembros de las comunidades indígenas de Laguna Blanca y Corral Blanco, sin ellos este trabajo no sería posible.

\section{REFERENCIAS}

Acosta Ochoa, G.

1999. Procesos de trabajo determinado. La configuración de los modos de trabajo en la cultura arqueológica. Boletín de Antropología Americana 35: 5-21.

Acosta Ochoa, G.

2011. Entre el lago y los volcanes. La cultura arqueológica asociada a la cerámica Azteca I. Imagina Digital. México.

Arnold. D.

1994. La tecnología cerámica andina: Una perspectiva etnoarqueológica. En Shimada, I. (ed.) Tecnología y organización de la producción cerámica prehispánica en los Andes: 477-504. Fondo Editorial, Pontificia Universidad Católica del Perú. Lima.
Balfet, H., M. Fauvet-Berthelot y S. Monzón. 1992. Normas para la descripción de vasijas cerámicas. CEMCA, México.

Bialakowsky, A.

2018. Vida cotidiana y reclasificaciones sociológicas según Giddens, Bourdieu, Habermas y Luhmann. Convergencia Revista de Ciencias Sociales 77.

https://doi.org/10.29101/crcs.v25i77.4456.

De La Fuente, G. A.

2011. Tinajas, ollas y yuros: producción de alfarería durante el período Tardío (ca. AD 900 - AD 1200) en Watungasta (Depto. Tinogasta, Catamarca, Argentina). En Páez, M. C. y G. A. De La Fuente (eds.) La cerámica arqueológica en la materialización de la sociedad. Transformaciones, 
metáforas y reproducción social: 87-100. BAR International Series 2294. Archaeopress. Oxford.

Delfino, D. D.

1999. Prospecciones en los '90: Nuevas evidencias para repensar la arqueología de Laguna Blanca (Dpto. Belén. Catamarca). Revista de Ciencia y Técnica 7: 55-80.

Delfino, D. D.

2005. Entre la dispersión y la periferia. Sentido de presencias. Lagunización de la aguada. En La Cultura de la aguada y sus expresiones regionales. Trabajos presentados en la $V$ Mesa redonda la cultura de la Aguada y su dispersión, Tomo I: 263291. Secretaría de Ciencia y Tecnología. Universidad Nacional de la Rioja.

Delfino, D., A. Díaz y V. Espiro.

2007. ¿Tierras vacas o complicidad administrativa? La reorientación económica del Bolsón puneño de Laguna Blanca a partir de la colonia. En Memorias del III Congreso de Historia de Catamarca, A. R. Bazán et al. (ed.). Tomo I: 107-124. Editorial Científica Universitaria. Catamarca.

Delfino, D. D., V. E. Espiro y R. A. Díaz.

2009. Modos de vida situados: El Formativo en Laguna Blanca. Andes 20: 111-134.

Delfino, D. D., V. E. Espiro y R. A. Díaz.

2010. Las pircas, los límites y sus entornos. Nuevas evidencias de la aldea arqueológica Laguna Blanca. En Actas del XVII Congreso Nacional de Arqueologia: Arqueología Argentina en el Bicentenario de la Revolución de Mayo, Bárcena, J. R. y H. Chiavazza (eds.), Tomo IV: 1667-1673. Zeta Editores. Mendoza.

Delfino, D., A. Díaz y V. Espiro.

2015. Prácticas sociales en el pasado y presente de Laguna Blanca (Dpto. Belén, Catamarca): reflexiones en torno al Modo de Vida Comunitario Agrocéntrico. En Korstanje, M. A., M. Lazzari, M. Basile, F. Bugliani, V. Lema, L. Pereyra Domingorena y M. Quesada (eds.) Crónicas materiales precolombinas: Arqueología de los primeros poblados del NO Argentino. Sección paisajes, territorios y redes de interacción: 385-426. Sociedad Argentina de Antropología. Buenos Aires.

Dobres, M. A.

2000. Technology and social agency. Blackwell. Londres.

Dobres A. y C. Hoffman.

1994. Social agency and the dinamics of Prehistoric technology. Journal of Archaoelogical Method and Theory 1 (3): 211-258.
Espiro, V. E.

2008. Características del proceso de manufactura de las alfarerías de la Aldea Piedra Negra, correspondientes al primer milenio de nuestra era, Distrito Laguna Blanca, Departamento Belén, Provincia de Catamarca. La Zaranda de Ideas, Revista de Jóvenes Investigadores en Arqueología 4: 9-25.

García Rosselló, J.

2009. Cadena operativa, forma, función y materias primas. Un aporte a través de la producción cerámica Mapuche. Relaciones de la Sociedad Argentina de Antropología 34: 123-148.

García Rosselló, J.

2011. Cerámica, prácticas, técnicas y estructura social mapuche: un caso de dinamismo cultural. Complutum 28 (2): 341-357.

Giddens, A.

2011. La constitución de la sociedad. Bases para una teoría de la estructuración. Amorrortu. Argentina.

Orton, C., P.Tyers y A. Vince.

1997. Pottery in Archaeology. University Press. Cambridge.

Padilla Fernández, J. J.

2017. Identidad y tecnología cerámica: el trabajo alfarero en Luxor (Egipto). Complutum 28 (2): 379398.

Rice, P.

1987. Pottery Analysis. University of Chicago Press. Chicago.

Rye, O.

1988. Pottery technology. Principles and reconstruction. Manuals on Archaeology 4. Australian National University. Taraxacum. Washington.

Varela Guarda, V.

2002. Enseñanzas de alfareros Toconceños: tradición y tecnología en la cerámica. Chungará 34 (2): 225-252.

Vargas Arenas, I.

1985. Modo de vida: categoría de las mediaciones entre formación social y cultural. Boletín de Antropología Americana 12: 6-16.

Veloz Maggiolo, M.

1984. La arqueología de la vida cotidiana: matices, historia y diferencias. Boletín de Antropología Americana 10: 5-21. 
Valeria Elizabeth Espiro 2021. Aproximación al trabajo alfarero e identidad en Laguna Blanca, Catamarca ...

Vidal, A. y J. García Rosselló.

2009. "Dime cómo lo haces": Una visión etnoarqueológica de las estrategias de aprendizaje en la alfarería tradicional. Arqueoweb 12. https://webs.ucm.es/info/arqueoweb/pdf/12/vidal.p df 